\title{
Hadronically decaying color-adjoint scalars at the LHC
}

\author{
Steffen Schumann, ${ }^{a, b}$ Adrien Renaud ${ }^{c}$ and Dirk Zerwas ${ }^{c}$ \\ ${ }^{a}$ Institut für Theoretische Physik, Universität Heidelberg, \\ 69120 Heidelberg, Germany \\ ${ }^{b}$ II. Physikalisches Institut, Universität Göttingen, \\ 3707r Göttingen, Germany \\ ${ }^{c} L A L$, IN2P3/CNRS, \\ Orsay, France \\ E-mail: s.schumann@thphys.uni-heidelberg.de, renaud@lal.in2p3.fr, \\ zerwas@lal . in2p3.fr
}

ABSTRACT: We study the phenomenology of the pair-production of scalar color-octet electroweak singlet states at the LHC. Such states appear in many extensions of the Standard Model. They can be pair-produced copiously at the LHC and will signal themselves as resonances in multijet final states. Beyond the QCD pair-production process we consider a vectorlike confinement scenario with an additional color-octet vector state. These vector particles can be produced in the s-channel and through their decay contribute to the scalar pair production. We point out the differences between the two hypotheses and device a strategy to distinguish them.

KEYwords: Phenomenological Models, Supersymmetry Phenomenology

ARXIV EPRINT: 1108.2957 


\section{Contents}

1 Introduction 1

2 Two benchmark models $\quad 2$

2.1 Hyper-pions in vectorlike confinement gauge theories 3

2.2 Sgluons in supersymmetric models with Dirac gauginos 4

2.3 Octet-scalar pair production 5

$\begin{array}{lll}3 & \text { Simulation of signal and background events } & 6\end{array}$

$\begin{array}{lll}4 & \text { Event selection and background estimation } & 7\end{array}$

$\begin{array}{llr}5 & \text { Results } & 8\end{array}$

$\begin{array}{llr}6 & \text { Conclusions } & 11\end{array}$

$\begin{array}{ll}\text { A Cross section ratio } & 12\end{array}$

\section{Introduction}

The potential to produce new colored particles is one of the most prominent features of hadron colliders such as the Fermilab Tevatron or the CERN LHC. There, new-physics states that directly couple to the partonic initial state can be produced copiously for a wide range of masses. Most candidate theories for physics beyond the Standard Model (SM) predict the occurrence of such new particles and there is a continuous effort to search for hints for their production. Recent examples include the search for supersymmetric squarks and gluinos [1-4] or lepto-quarks [5, 6]. The various searches might be classified according to the considered final states. Besides a certain number of hadronic jets, remnants of the particle's color charge, this includes identified leptons from electroweak decays, associated photons and/or missing transverse energy from undetected decay products, e.g. a potential dark matter particle.

In this paper we analyse the pair-production process for new scalar color-octet states that are singlets under the electroweak gauge group. Such states appear in various extensions of the SM either as new fundamental particles [7-9] or composite objects [10,11]. As we consider states in the color-octet representation their QCD production cross section is fixed. The pair-production process, however, might have contributions depending on the theory that embeds the new scalars such as additional new particles that are produced resonantly in the $s$-channel and can decay into a pair of such octet scalars. The decays of the new scalar particles are of course highly model dependent and closely related to the underlying theory's spectrum. However, most likely, for a certain mass range two-body 
decays to SM partons will dominate and it is this decay mode we focus on here. As a consequence we have to search for the states under question in multijet final states, where they would signal themselves as dijet resonances.

The precise signature for the hadronically decaying resonances certainly depends on the mass of the new states as well as the experimental trigger requirements. The continuum QCD production process will favour pair production at the kinematic threshold. In consequence we consider inclusive four-jet production as the search environment. In situations where the octets get produced with sufficiently large transverse momentum a dedicated subjet analysis in dijet final states might be the most powerful search strategy [12].

The central challenge of the analysis is to dig up the signal from the enormous QCD multijet background that exceeds the signal by orders of magnitude. The key point is to make optimal use of the kinematics of the signal events to represent the production of two equal mass narrow scalar resonances. In an earlier study the feasibility of this analysis was shown for the vectorlike confinement scenario [11]. Here we further improve that analysis, extend it to discovering the pure QCD pair-production process, and point out how different models could be differentiated experimentally. We propose a data-driven approach to extract the relevant QCD multijet background, thereby minimizing the dependency on theoretical predictions.

In principle our candidate particles can also be produced singly at hadron colliders and there exist very stringent bounds from dijet resonance searches. For example ref. [13] quotes a $95 \% \mathrm{CL}$ exclusion limit of $1.91 \mathrm{TeV}$ for a color-octet scalar resonance with an order unity coupling to two gluons [14]. However, as soon as this interaction is induced at the loop-level only, the single-production rate drops significantly thus evading the strong limit. Such scenarios we want to consider here, for which the pair-production process is the most promising discovery channel.

The paper is organized as follows. In section 2 we define our two benchmark scenarios, namely the most simple vectorlike confinement model and the SM extension by a complex scalar gluon that decays into two gluons with probability one. In section 3 we describe the tools used to simulate signal and background events. In section 4 we give details on our event-selection criteria and discuss a method that allows an extraction of the QCD background from data. In section 5 we present results for our proposed search showing how one could potentially distinguish between the two benchmark models. Section 6 contains our conclusions.

\section{Two benchmark models}

Throughout this paper we want to consider two benchmark models for the production of electroweak singlet color-octet states. The first one is a vectorlike confinement scenario featuring besides our scalar candidate, the real octet hyper-pion, a further vector resonance in the color adjoint, called hyper-rho or coloron, that predominantly decays into a pair of hyper-pions. As the second template we consider the scalar partners of the gluino (called sgluons) as they appear in extended versions of the Minimal Supersymmetric Standard Model, such as the Minimal $R$-symmetric Supersymmetric Standard Model (MRSSM) [15] 
or an $N=1 / N=2$ hybrid model of supersymmetry with the $N=2$ supersymmetry implemented only in the gaugino sector [9]. In the following we will briefly define our two model hypotheses and discuss the different predictions for the pair-production rate of the two scalar octet candidates, the hyper-pion and the sgluon respectively.

\subsection{Hyper-pions in vectorlike confinement gauge theories}

The approach of vectorlike confining gauge theories at the TeV scale, first proposed in [16] and later on generalized in $[17,18]$, provides an attractive ansatz for potential physics beyond the SM. Assuming a minimal extension of the SM by new matter fields and making use of a fundamental mechanism already realized in nature, namely a confining strong force, a very rich phenomenology can be obtained from those theories described through rather few parameters only. The starting point is a quite minimal extension of the SM by new fermions in vectorlike representations of the SM gauge groups. These new fermions, charged under the strong and/or electroweak gauge group, feel a new strong gauge force called hypercolor (HC) that confines at the TeV scale, just as QCD confines at scales $\mathcal{O}(100 \mathrm{MeV})$. The emerging hyper-hadrons can be produced at the LHC signalling themselves through their decays mediated by hypercolor or SM interactions. The LHC phenomenology of vectorlike confinement will be dominated by the production of the lightest scalar and vector states, namely the pseudo-Nambu-Goldstone bosons called hyper-pions $\tilde{\pi}$ and the counterpart of the QCD $\rho$ meson, the hyper-rho $\tilde{\rho}$.

We want to examine the most minimal vectorlike-confinement model that has been studied before in refs. $[11,16,19,20]$. We consider three new massless vectorlike fermions charged under QCD and hypercolor only. The hypercolor gauge group is fixed to $\mathrm{SU}(3)_{\mathrm{HC}}$ and we assign the fermions as bi-fundamentals of the $\mathrm{SU}(3)_{\mathrm{c}} \otimes \mathrm{SU}(3)_{\mathrm{HC}}$ product group and singlets under the electroweak interactions. The lowest lying states below the hypercolorconfinement scale $\Lambda_{H C} \sim \mathrm{TeV}$ are the color-adjoint pseudo-Nambu-Goldstone hyper-pions $\tilde{\pi}$ and the octet hyper-rho or coloron $\tilde{\rho}$. For this scaled-up version of QCD their mass ratio is approximated by

$$
\frac{m_{\tilde{\pi}}}{m_{\tilde{\rho}}} \simeq 0.3
$$

with $m_{\tilde{\rho}} \sim \Lambda_{H C}$. The effective Lagrangian describing the dynamics and interactions of the hyper-pion and hyper-rho is given by [11]

$$
\begin{aligned}
& \mathcal{L}_{\mathrm{eff}}^{\mathrm{HC}}=-\frac{1}{4} G_{\mu \nu}^{a} G^{a \mu \nu}-\frac{1}{4} \tilde{\rho}_{\mu \nu}^{a} \tilde{\rho}^{a \mu \nu}+\frac{m_{\tilde{\rho}}^{2}}{2} \tilde{\rho}_{\mu}^{a} \tilde{\rho}^{a \mu}+\bar{q} i \gamma^{\mu}\left(\partial_{\mu}+i g_{3}\left(G_{\mu}+\varepsilon \tilde{\rho}_{\mu}\right)\right) q \\
& +\frac{1}{2}\left(D_{\mu} \tilde{\pi}\right)^{a}\left(D^{\mu} \tilde{\pi}\right)^{a}-\frac{m_{\tilde{\pi}}^{2}}{2} \tilde{\pi}^{a} \tilde{\pi}^{a}-g_{\tilde{\rho} \tilde{\pi} \tilde{\pi}} f^{a b c} \tilde{\rho}_{\mu}^{a} \tilde{\pi}^{b} \partial^{\mu} \tilde{\pi}^{c}-\frac{3 g_{3}^{2}}{16 \pi^{2} f_{\tilde{\pi}}} \operatorname{Tr}\left[\tilde{\pi} G_{\mu \nu} \widetilde{G}^{\mu \nu}\right] \\
& +\xi \frac{2 i \alpha_{s} \sqrt{3}}{m_{\tilde{\rho}}^{2}} \operatorname{Tr}\left(\tilde{\rho}_{\nu}^{\mu}\left[G_{\sigma}^{\nu}, G_{\mu}^{\sigma}\right]\right)+i \chi g_{3} \operatorname{Tr}\left(G_{\mu \nu}\left[\tilde{\rho}^{\mu}, \tilde{\rho}^{\nu}\right]\right) .
\end{aligned}
$$

The first line contains the SM and hyper-rho kinetic terms and the coupling of the hyper-rho to quarks induced by kinetic mixing of the gluon and coloron fields. The second line summarises the kinetic and mass terms for the hyper-pion and the effective vertices 

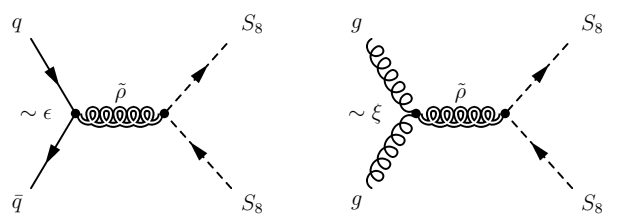

Figure 1. Feynman diagrams for resonant coloron production decaying into a pair of color-adjoint scalars. Note that we consider $\epsilon=0.2$ while we set $\xi=0$ such that the gluon-gluon fusion contribution vanishes.

that describe the decays $\tilde{\rho} \rightarrow \tilde{\pi} \tilde{\pi}$ and $\tilde{\pi} \rightarrow g g$. The terms in the third line represent strong interaction matrix elements of the underlying theory that cannot be extracted from the QCD analog [11]. The parameter $\xi$ is an undetermined number of order one. We use the most conservative assumption of $\xi=0$ as nonzero values enhance the pion pair-production cross section, cf. ref. [11], through an additional resonant coloron contribution in the gluongluon fusion channel, cf. figure 1. The term proportional to $\chi$ is of no relevance for our analysis as it contributes to the coloron pair-production process only [11].

All other parameters of the model are fixed by just scaling up the QCD spectrum and the corresponding couplings of the low-energy QCD chiral Lagrangian [16]. The mixing parameter $\epsilon$ is determined to $\epsilon \simeq 0.2$ and the coloron to hyper-pion coupling is given by $g_{\tilde{\rho} \tilde{\pi} \tilde{\pi}} \simeq 6$. The hyper-pion decay constant is determined through

$$
\frac{f_{\tilde{\pi}}}{\Lambda_{\mathrm{HC}}} \simeq \frac{f_{\pi}}{\Lambda_{\mathrm{QCD}}}
$$

accordingly the hyper-pion decays promptly into two gluons. The coloron is rather broad state,

$$
\frac{\Gamma_{\tilde{\rho}}}{m_{\tilde{\rho}}} \approx 0.19
$$

Its dominant decay mode however is not into dijets but rather a pair of hyper-pions, determined by

$$
\frac{\Gamma_{\tilde{\rho} \rightarrow q \bar{q}}}{\Gamma_{\tilde{\rho} \rightarrow \tilde{\pi} \tilde{\pi}}} \sim \frac{\epsilon}{g_{\tilde{\rho} \tilde{\pi} \tilde{\pi}}} .
$$

\subsection{Sgluons in supersymmetric models with Dirac gauginos}

Supersymmetric models that accomplish Dirac gauginos provide an attractive solution to the flavour problem of the MSSM [15]. This is achieved through an additional suppression of dangerous flavour-violating processes. Considering a Dirac gluino, in order to account for its four degrees of freedom, besides the gluon two additional bosonic degrees of freedom must be added in a viable supersymmetric theory. This complex color-adjoint scalar, known as the sgluon, has explicit realisations in the context of the MRSSM [8] or a phenomenological $N=1 / N=2$ SUSY hybrid model as considered in [9]. 

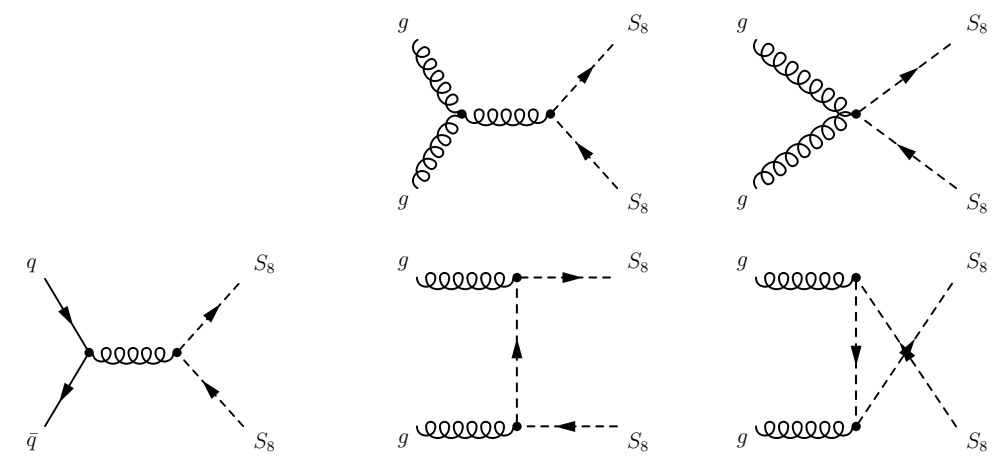

Figure 2. Feynman diagrams for the QCD pair production of color adjoints in the $q \bar{q}$ and $g g$ channel.

The sgluon has a gauge coupling to gluons and its pair-production rate is thus given by QCD. It interacts with quarks only at the one-loop level with the couplings being proportional to the quark masses. The sgluon-gluon-gluon interaction also occurs at the one-loop level only and is mediated by squarks. Accordingly resonant single-sgluon production is strongly suppressed. The sgluon coupling to two gluinos is the supersymmetric partner of the gluon coupling, while the coupling to squarks originates from $D$ terms and is proportional to the Dirac gluino mass [8].

The best laboratory to discover the sgluon is its pair-production process. In [8] two split sgluon states have been considered. The work focused on the spectacular decay of the sgluon into top or anti-top accompanied by a light-flavour quark what leads to a jetassociated same-sign top signature. If heavy enough the sgluon can decay at the tree-level into pairs of gluinos or squarks. It is those decays that ref. [9] focused on along with the decay into $t \bar{t}$, however, the authors considered a degenerate pair of sgluons.

In both scenarios the sgluon has a sizeable branching fraction into gluons that dominates certainly below the top threshold. It is this part of the sgluon phenomenology we want to study here. To be precise, we consider two degenerate sgluon states as in ref. [9] and fix the sgluon branching ratio to gluons to unity independent of its actual mass. Such we simplify the underlying extended supersymmetric model but we still capture a large part of its phenomenology. In order to establish the supersymmetric theory that embeds the scalar adjoint the decay channels discussed in refs. $[8,9]$ need to be studied.

\subsection{Octet-scalar pair production}

In figure 2 we depict the Feynman diagrams for the QCD pair production of hyper-pions and sgluons from $q \bar{q}$ and $g g$ initial states. The way we set up our sgluon benchmark model its pair-production rate exceeds the continuum QCD production rate for a pair of hyper-pions by a factor of two, consequence of the degeneracy of the two sgluon states. However, the actual hyper-pion pair-production rate is determined by the additional $s$ channel coloron contributions depicted in figure 1 . In figure 3 (Left) we compare the $7 \mathrm{TeV}$ LHC cross sections for the hyper-pion and the sgluon pair-production signal as a function of the scalar mass. The ratio of the two cross sections is shown in figure 3 (Right) as function 

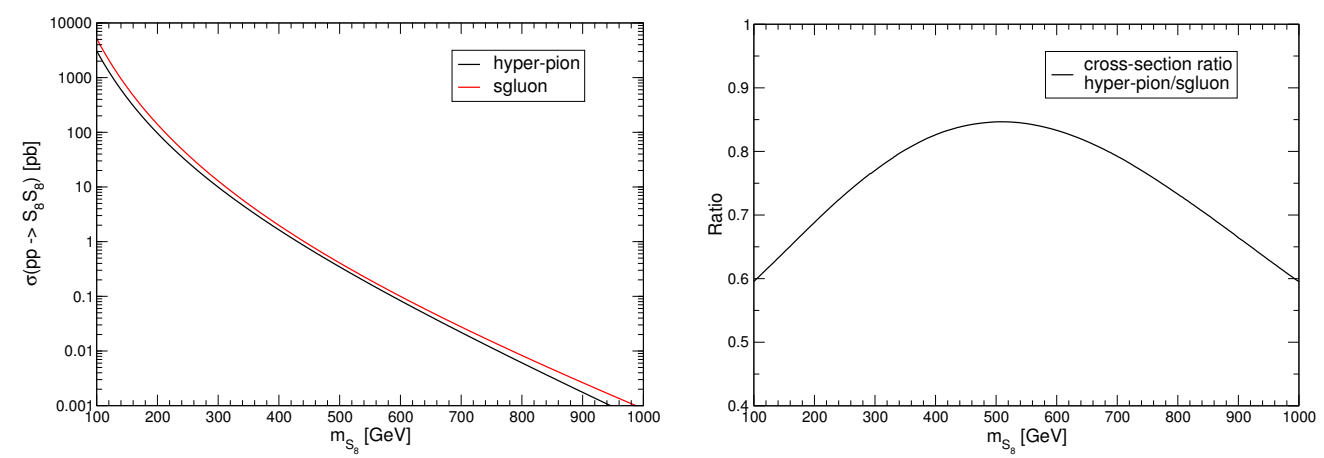

Figure 3. (Left) The tree-level total cross sections at the $7 \mathrm{TeV}$ LHC for the production of a pair of octet scalars. Besides the hyper-pion signal we present the cross section for producing a pair of scalar gluons $[8,9]$. (Right) The ratio of the hyper-pion and sgluon cross section as function of the scalar mass.

of the mass. For low scalar masses the ratio is almost 0.6 as expected for a real scalar with respect to a complex scalar field. At higher masses the ratio approaches 0.83 as the contribution of the $s$-channel coloron exchange becomes more important before dropping back to 0.6 at $1 \mathrm{TeV}$.

The two models outlined above shall serve as concrete realisations of new color-adjoint scalars. As mentioned before such particles appear in many Standard Model extensions. The pair production of electroweak singlets decaying into heavy quarks has for example been considered in refs. [21-23], rare decays have been studied in ref. [24], phenomenological analyses of electroweak-charged adjoint scalar production can be found in refs. [25-27].

\section{Simulation of signal and background events}

Before turning into the discussion of our search strategy and signal selection criteria we want to briefly describe the simulation tools employed to model the signal and background processes.

We use the SHERPA event generator [28] to simulate both, the hyper-pion and sgluon pair-production signal as well as the QCD multijet background. The signal models have been implemented in the matrix-element generator AMEGIC++ [29]. The Feynman rules for the hyper-color model have been derived from eq. (2.2). The interactions relevant for the pair-production of sgluons are given by QCD and we assume the sgluon branching ratio to gluons equal to unity.

For the signal events we set the factorisation and renormalisation scales equal to the scalars mass, i.e. $\mu_{F}=\mu_{R}=m_{S_{8}}^{2}$. The decay of the scalar resonances into gluons we treat in the narrow-width-approximation, whereas we fully take into account off-shell effects for the broad coloron $s$-channel contribution in the hyper-color scenario. The parton-level signal events then get passed to SHERPA's parton-shower and hadronisation routines $[28,30]$ in order to obtain realistic particle-level events.

The modelling of the QCD multijet backgrounds is a severe challenge [31]. Up to now the next-to-leading order corrections to four-jet production are unknown so we have to rely 
on leading-order predictions. To accurately simulate multijet production we employ matrixelement parton-shower matching as implemented in SHERPA [32, 33]. We consider the complete sets of tree-level matrix elements with up to six final-state partons from SHERPA's matrix-element generator COMIX [34]. The matrix elements of varying multiplicity get consistently combined to an inclusive sample of fully exclusive events through subsequent parton-shower evolution [30] before subject to hadronisation. The renormalisation and factorisation scales are dynamically determined on an event-by-event basis according to the matching algorithm [32]. For the parton-separation parameter of the matching procedure we use $Q_{\text {cut }}=30 \mathrm{GeV}$. Note that SHERPA's model for QCD multijet production has already successfully been validated against data from HERA [35], Tevatron [36, 37] and the LHC [38, 39], what makes us confident to use a realistic background estimate throughout this study. In section 4 we describe a strategy to extract the normalisation and shape of the QCD background in a data-driven approach.

To account for detector-resolution effects such as finite granularity and jet smearing we process the particle-level signal- and background events through the publically available detector-simulation package DelPhes [40]. DelPhES yields a reliable simulation for a prototypical LHC detector that has been used in several physics studies such as [41-43]. For defining jets we make use of the FASTJET [44] package.

\section{Event selection and background estimation}

Throughout the analysis we use anti- $k_{T}$ jets [45] with an $R$ parameter of 0.6. Events are required to have at least four jets with a minimal $p_{T}$ of $50 \%$ of the mass of the resonance. Once this $p_{T}$ selection is applied the signal is composed of octet scalars produced with a non-negligible transverse boost. In the $\eta-\phi$ plane the separation of the gluon jets from the decay is typically $\Delta R \approx 1$. We then consider all the possible pairings between the four highest- $p_{T}$ jets and retain the combination that minimizes $\left|\Delta R_{i j}-1\right|+\left|\Delta R_{k l}-1\right|$ where $i, j, k, l$ denote the four leading jets. If one of the reconstructed resonances has a jet separation larger than $\Delta R_{i j}=1.6$, the event is rejected.

To improve further the rejection of the background, the relative difference between the two reconstructed masses is required to agree within $7.5 \%$, i.e. $\left|M_{1}-M_{2}\right| /\left(M_{1}+M_{2}\right)<$ 0.075. Additional separation between background and signal is obtained by using the reconstructed scattering angle $\left(\cos \left(\theta^{*}\right)\right)$ in the center-of-mass frame of the four jets to be less than 0.6. The $\cos \left(\theta^{*}\right)$ distribution is shown in figure 4 for the hyper-pion signal and the QCD background. All cuts but the cut on the scattering angle were applied. For the signal the distribution is $\sin \left(2 \theta^{*}\right)$ like as the production of scalar particles is central, whereas for the QCD background the production in the forward region is more pronounced.

The mean of the two reconstructed masses for events that pass all cuts is shown in figure 5 for a hyper-pion signal of $m_{\tilde{\pi}}=100 \mathrm{GeV}$. The data corresponds to an integrated luminosity of $1 \mathrm{fb}^{-1}$ at the $7 \mathrm{TeV}$ LHC. As the form of the signal is similar to the form of the background, the ABCD matrix method is used to predict the background. The four regions are separated via the scattering angle (less than or greater than 0.6) and the relative mass difference (inverting the cut described above), cf. figure 6 . The region with 


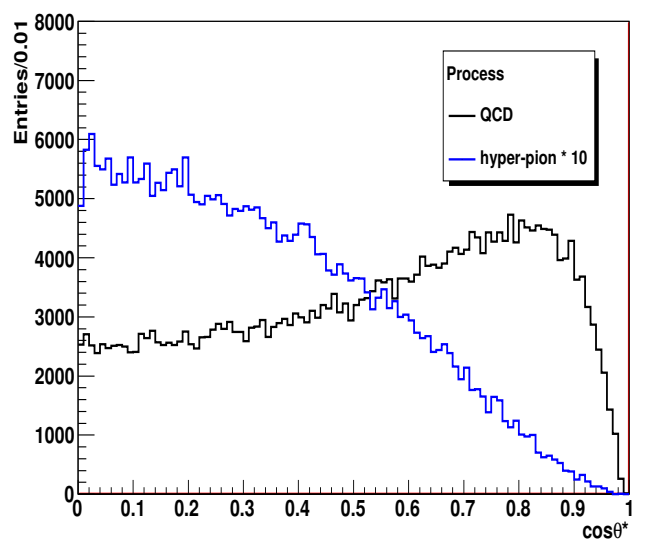

Figure 4. Distribution of the reconstructed scattering angle in the center-of-mass frame of the reconstructed four highest- $p_{T}$ jets. Note, for this shape comparison the signal was scaled by a factor of 10 .

the highest signal purity for that we want to estimate the background normalisation and shape is denoted as A. Under the assumption our two discriminators are uncorrelated for the QCD background, we can estimate the number of background events in region A to

$$
N_{\mathrm{BG}}^{A}=\frac{N_{\mathrm{BG}}^{B} N_{\mathrm{BG}}^{D}}{N_{\mathrm{BG}}^{C}} .
$$

The background shape we take from region $D$, where only the cut on the scattering angle is reversed with respect to the signal selection. The background prediction from the ABCD method is shown in figure 5. Both the background shape as well as the normalisation are predicted well when comparing to the Monte-Carlo truth. This data driven approach will have to be validated with actual collision data of course, i.e., will the same correlation pattern be observed in the data as predicted by Monte Carlo and which scale factor will have to be applied to the Monte-Carlo prediction to account for higher-order corrections. Here our emphasis was on the background per se, in the actual data potential signal contaminations in regions $B, C$ and $D$ will have to be taken into account as well.

\section{Results}

In figure 7 the cross sections after all cuts are shown as function of the scalar mass. The QCD background decreases strongly as expected.

In figure 8 the sensitivity of the analysis is shown as function of the hyper-pion mass. We define the sensitivity as the statistical error on the background (and its prediction) for $1 \mathrm{fb}^{-1}$ in a window of $40 \mathrm{GeV}$ centered on the nominal hyper-pion mass divided by the efficiency and multiplied by 1.64 (similar to a one-sided Gaussian counting experiment limit).

As the instantaneous luminosity increases the trigger thresholds of the experiments will increase, using as we are using a sliding $p_{T}$ cut, cf. section 4 , the analysis is valid for masses greater than twice the trigger threshold. Lower masses than that are not accessible. 


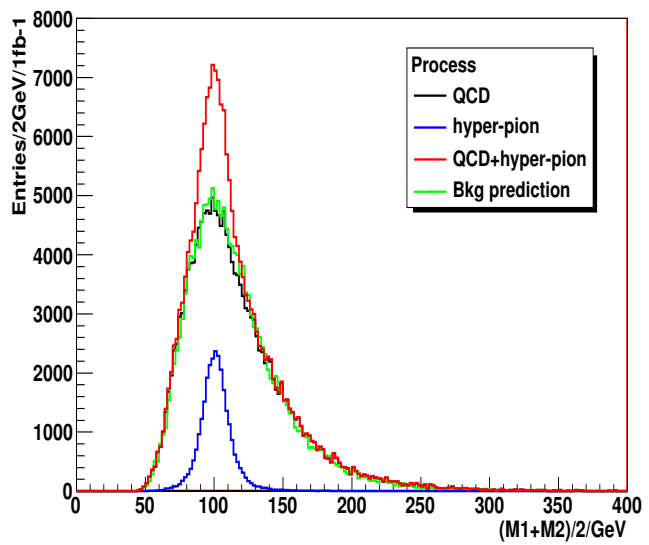

Figure 5. The average mass for a hyper-pion signal of $m_{\tilde{\pi}}=100 \mathrm{GeV}$, the QCD background and the sum of signal plus background are shown after all cuts for an integrated luminosity of $1 \mathrm{fb}^{-1}$. The green line is the predicted background according to the ABCD method.

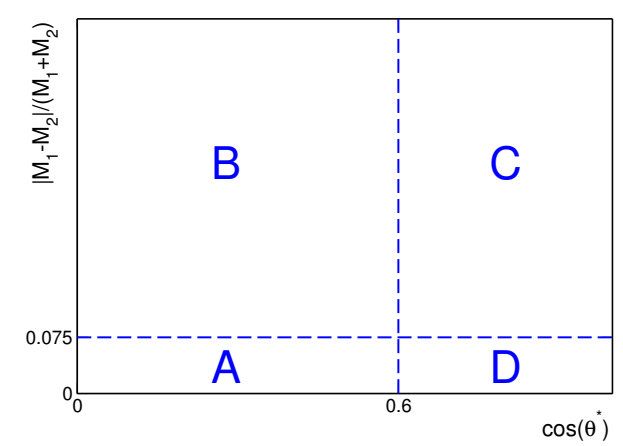

Figure 6. The four event categories used in the ABCD method. The two discriminators are the scattering angle $\cos \left(\theta^{*}\right)$ and the relative mass difference of the two reconstructed resonance candidates. The signal-enriched region corresponds to selection A.

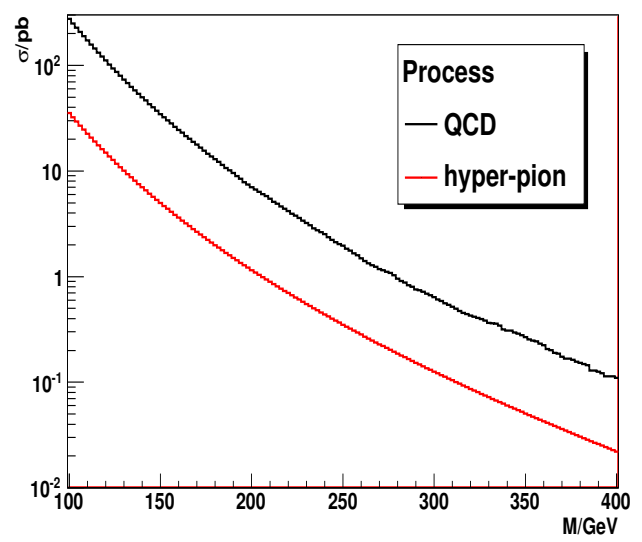

Figure 7. Cross sections after all cuts for the QCD background, and the hyper-pion signal. 


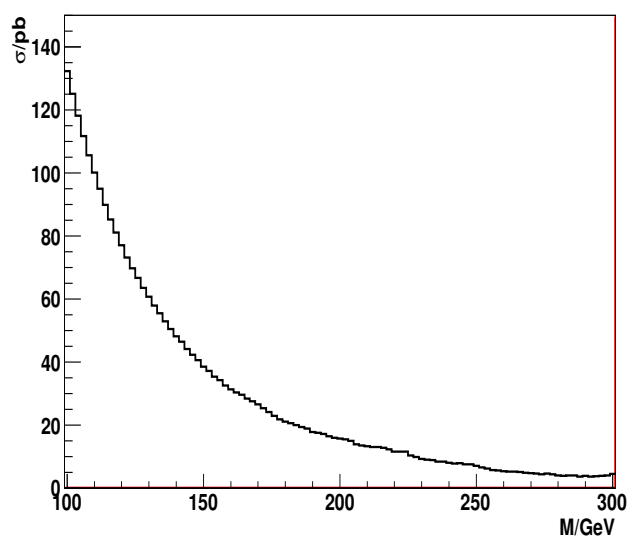

Figure 8. The sensitivity of the analysis is shown, based on the statistical error in a window of $40 \mathrm{GeV}$ centered on the nominal mass.

\begin{tabular}{|lcccc|}
\hline & $100 \mathrm{GeV}$ & $225 \mathrm{GeV}$ & $300 \mathrm{GeV}$ & $500 \mathrm{GeV}$ \\
\hline hyper-pion & $0.9 \%$ & $1.1 \%$ & $1.2 \%$ & $1.2 \%$ \\
sgluon & $0.6 \%$ & $0.4 \%$ & $0.3 \%$ & $0.2 \%$ \\
\hline
\end{tabular}

Table 1. Efficiencies are shown as function of the scalar mass.

For masses far from the trigger threshold one could perform a sideband analysis only in the signal region (bump hunting), i.e., an analysis with a fixed cut on the transverse momentum. This however can lead to double peak structures for the signal, diluting somewhat the gain on the efficiency.

For a mass of $300 \mathrm{GeV}$ the hyper-pion (sgluon) cross section is $10 \mathrm{pb}(13 \mathrm{pb}$ ). The sensitivity of the analysis is $5 \mathrm{pb}$. Thus even adding experimental errors, the unknown K-factor of the background cross section, we can surely state that the four-jet final state can be probed in spite of the huge QCD background.

The difference between the hyper-pion and sgluon pair production goes beyond a scaling of the cross sections. The $s$-channel coloron exchange also has an impact on the kinematics and therefore on the selection efficiency. In figure 9 the transverse-momentum distribution of the 4 th jet is shown for a signal mass of $300 \mathrm{GeV}$. The $s$-channel coloron increases the $p_{T}$ and therefore for the same analysis the efficiency for hyper-pions and sgluons is not the same as shown in table 1. While the efficiency for the hyper-pions is stable, the efficiency for sgluons decreases slowly for increasing masses. It is interesting to note, that the efficiency as a function of the $p_{T}$ cut behaves differently in the two scenarios. For example for a scalar mass of $500 \mathrm{GeV}$ a decrease of the $p_{T}$ cut by $20 \%$ leads to an increase of the hyper-pion selection efficiency by a factor of 2 , the efficiency of the sgluon signal, however, increases by a factor of 3. A dedicated additional analysis for masses far from the trigger threshold could be envisaged that could provide further information to discriminate the two models.

An interesting question arises also whether the coloron mass can be reconstructed. To address this question we consider the invariant mass of the two reconstructed scalar 


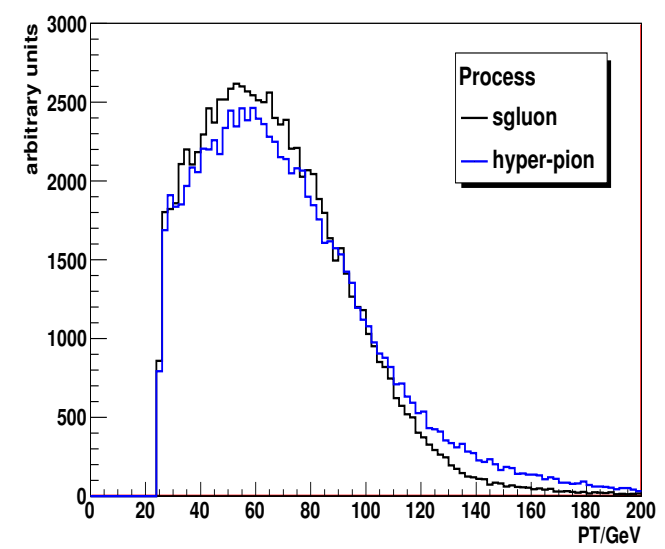

Figure 9. The distribution of the $4^{\text {th }}$ jet is shown for the sgluon and hyper-pion signal for a scalar mass of $300 \mathrm{GeV}$ (before cuts).

candidates. As can be seen in figure 10 at low mass the coloron mass distribution is diluted by the non-resonant production mechanism. No clear distinction can be observed between the sgluon and hyper-pion case. However for masses of $300 \mathrm{GeV}$ as well as $500 \mathrm{GeV}$, cf. the middle and right panel of figure 10, the coloron of the hyper-pion model becomes visible, thus giving us possibly a handle to distinguish the two models. We note however that in order to differentiate the two hypotheses, the actual QCD background (larger than the signal) will have to be dealt with. While the signal differences are quite striking, an integrated luminosity much larger than $1 \mathrm{fb}^{-1}$ will be necessary to conclude on the underlying model and only if the signal turns out to be at sufficiently high mass. However, the simulation of such large integrated luminosities for the background is beyond the scope of our paper. Furthermore we want to add that for an LHC center-of-mass energy of $14 \mathrm{TeV}$ the discrimination of the two models through the reconstruction of the coloron resonance gets more complicated due to the different initial-state parton fluxes. For the hyper-pion mass range considered here, namely $m_{\tilde{\pi}}=100 \ldots 500 \mathrm{GeV}$, at $14 \mathrm{TeV}$ the hyper-pion to sgluon cross-section ratio is strictly smaller than for $7 \mathrm{TeV}$. In turn the relative importance of the coloron contribution to the total hyper-pion pair-production cross section is largely reduced and the QCD continuum production mechanism dominates. On the other hand this dependence on the collision energy might serve as a handle to establish the coloron production from $q \bar{q}$ initial states.

The alternative way to verify the existence of the hyper-rho state is to study its pairproduction process. In refs. $[11,19,20]$ the signature $p p \rightarrow \tilde{\rho} \tilde{\rho} \rightarrow 4 \tilde{\pi} \rightarrow 8$ jets has been studied and it was shown that the LHC provides great discovery potential both at 7 and $14 \mathrm{TeV}$ center-of-mass energy.

\section{Conclusions}

We have presented a search strategy for the pair production of new color-adjoint scalar particles that decay into pairs of jets. Such states are predicted by many extensions of 

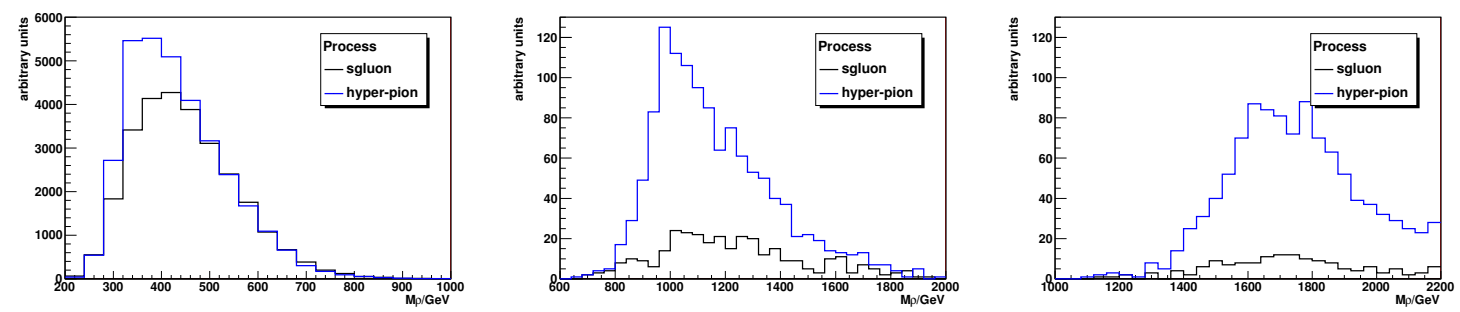

Figure 10. The distribution of the reconstructed coloron mass is shown after all cuts for the hyper-pion model and the sgluon model. For the latter the distribution is flatter, whereas for the former the $s$-channel contribution is visible. From left to right the distributions correspond to scalar masses of 100,300 and $500 \mathrm{GeV}$. In the hyper-color scenario the corresponding coloron masses are 333, 1000 and $1667 \mathrm{GeV}$, respectively.

the Standard Model. New color octets can be produced in abundance at the LHC and their production rate is rather model independent. We considered two benchmark models as predictive realisations, a simplified sgluon model and a vectorlike confinement scenario that features an additional vector resonance in the color adjoint.

While the QCD backgrounds seem overwhelming at first sight, the use of an equalmass constraint for the two reconstructed dijet resonances and the angular distribution of the production of the scalars help to reduce the backgrounds to a manageable level. Both models are clearly detectable at the LHC for a broad range of masses. When searching for scalar octets with $m_{S_{8}} \gtrsim 300 \mathrm{GeV}$ such that $m_{S_{8}} / 2$ is well above the trigger threshold the analysis cuts might be adjusted to further optimise the signal yield and increase statistics. Comparing the two benchmark models for different masses at low mass only the cross section provides some differentiation. If the scalars have higher mass, the reconstruction of the four-jet invariant mass might shed light on the presence of an additional $s$-channel resonance decaying into a pair of scalars, however an integrated luminosity bigger than $1 \mathrm{fb}^{-1}$ is needed.

\section{Acknowledgments}

We thank Peter Zerwas for helpful discussions on the sgluon model. SS would like to thank Can Kiliç for fruitful discussions and Elmar Bittner for computing support. Most simulations underlying this study have been performed on bwGRiD (http://www. bw-grid.de), member of the German D-Grid initiative, funded by the Ministry for Education and Research and the Ministry for Science, Research and Arts Baden-Württemberg. Part of these studies were performed in the context of the GDR Terascale (CNRS).

\section{A Cross section ratio}

The ratio of the cross sections for the pair production of hyper-pions and sgluons is given in table 2. The calculation was performed at leading order using the CTEQ6L1 PDFs [46]. 


\begin{tabular}{|cc|cc|cc|cc|}
\hline $\mathrm{M} / \mathrm{GeV}$ & & $\mathrm{M} / \mathrm{GeV}$ & & $\mathrm{M} / \mathrm{GeV}$ & & $\mathrm{M} / \mathrm{GeV}$ & \\
100 & 0.6 & 330 & 0.79 & 560 & 0.84 & 790 & 0.74 \\
110 & 0.6 & 340 & 0.8 & 570 & 0.84 & 800 & 0.73 \\
120 & 0.61 & 350 & 0.8 & 580 & 0.84 & 810 & 0.73 \\
130 & 0.62 & 360 & 0.81 & 590 & 0.84 & 820 & 0.72 \\
140 & 0.63 & 370 & 0.81 & 600 & 0.83 & 830 & 0.71 \\
150 & 0.64 & 380 & 0.82 & 610 & 0.83 & 840 & 0.71 \\
160 & 0.65 & 390 & 0.82 & 620 & 0.83 & 850 & 0.7 \\
170 & 0.66 & 400 & 0.83 & 630 & 0.82 & 860 & 0.69 \\
180 & 0.67 & 410 & 0.83 & 640 & 0.82 & 870 & 0.69 \\
190 & 0.68 & 420 & 0.83 & 650 & 0.82 & 880 & 0.68 \\
200 & 0.69 & 430 & 0.84 & 660 & 0.81 & 890 & 0.67 \\
210 & 0.7 & 440 & 0.84 & 670 & 0.81 & 900 & 0.66 \\
220 & 0.71 & 450 & 0.84 & 680 & 0.8 & 910 & 0.66 \\
230 & 0.72 & 460 & 0.84 & 690 & 0.8 & 920 & 0.65 \\
240 & 0.72 & 470 & 0.84 & 700 & 0.79 & 930 & 0.64 \\
250 & 0.73 & 480 & 0.84 & 710 & 0.79 & 940 & 0.64 \\
260 & 0.74 & 490 & 0.85 & 720 & 0.78 & 950 & 0.63 \\
270 & 0.75 & 500 & 0.85 & 730 & 0.78 & 960 & 0.62 \\
280 & 0.76 & 510 & 0.85 & 740 & 0.77 & 970 & 0.62 \\
290 & 0.76 & 520 & 0.85 & 750 & 0.76 & 980 & 0.61 \\
300 & 0.77 & 530 & 0.85 & 760 & 0.76 & 990 & 0.6 \\
310 & 0.78 & 540 & 0.85 & 770 & 0.75 & 1000 & 0.6 \\
320 & 0.78 & 550 & 0.84 & 780 & 0.75 & & \\
\hline
\end{tabular}

Table 2. Ratio of the hyper-pion cross section to the sgluon cross section.

Open Access. This article is distributed under the terms of the Creative Commons Attribution Noncommercial License which permits any noncommercial use, distribution, and reproduction in any medium, provided the original author(s) and source are credited.

\section{References}

[1] CMS collaboration, V. Khachatryan et al., Search for supersymmetry in pp collisions at $7 \mathrm{TeV}$ in events with jets and missing transverse energy, Phys. Lett. B 698 (2011) 196 [arXiv:1101.1628] [SPIRES].

[2] ATLAS collaboration, J.B.G. da Costa et al., Search for squarks and gluinos using final states with jets and missing transverse momentum with the ATLAS detector in $\sqrt{s}=7 \mathrm{TeV}$ proton-proton collisions, Phys. Lett. B 701 (2011) 186 [arXiv:1102.5290] [SPIRES].

[3] ATLAS collaboration, G. Aad et al., Search for supersymmetry using final states with one lepton, jets and missing transverse momentum with the ATLAS detector in $\sqrt{s}=7 \mathrm{TeV} p p$, Phys. Rev. Lett. 106 (2011) 131802 [arXiv:1102.2357] [SPIRES]. 
[4] CMS collaboration, S. Chatrchyan et al., Search for supersymmetry in pp collisions at $\sqrt{s}=7$ TeV in events with a single lepton, jets and missing transverse momentum, JHEP 08 (2011) 156 [arXiv:1107.1870] [SPIRES].

[5] CMS collaboration, V. Khachatryan et al., Search for pair production of first-generation scalar leptoquarks in pp collisions at $\sqrt{s}=7$ TeV, Phys. Rev. Lett. 106 (2011) 201802 [arXiv: 1012.4031] [SPIRES].

[6] ATLAS collaboration, G. Aad et al., Search for pair production of first or second generation leptoquarks in proton-proton collisions at $\sqrt{s}=7$ TeV using the ATLAS detector at the LHC, arXiv: 1104.4481 [SPIRES].

[7] G. Burdman, B.A. Dobrescu and E. Ponton, Resonances from two universal extra dimensions, Phys. Rev. D 74 (2006) 075008 [hep-ph/0601186] [SPIRES].

[8] T. Plehn and T.M.P. Tait, Seeking sgluons, J. Phys. G 36 (2009) 075001 [arXiv:0810. 3919] [SPIRES].

[9] S.Y. Choi et al., Color-octet scalars of $N=2$ supersymmetry at the LHC, Phys. Lett. B 672 (2009) 246 [arXiv:0812.3586] [SPIRES].

[10] C.T. Hill and E.H. Simmons, Strong dynamics and electroweak symmetry breaking, Phys. Rept. 381 (2003) 235 [Erratum ibid. 390 (2004) 553] [hep-ph/0203079].

[11] C. Kilic, S. Schumann and M. Son, Searching for multijet resonances at the LHC, JHEP 04 (2009) 128 [arXiv:0810.5542] [SPIRES].

[12] Y. Bai and J. Shelton, Composite octet searches with jet substructure, arXiv:1107.3563 [SPIRES].

[13] ATLAS collaboration, Search for new physics in dijet mass distributions in $0.81 \mathrm{fb}^{-1}$ of $\mathrm{pp}$ collisions at $\sqrt{s}=7$ TeV, ATLAS-CONF-2011-095 (2011).

[14] T. Han, I. Lewis and Z. Liu, Colored resonant signals at the LHC: largest rate and simplest topology, JHEP 12 (2010) 085 [arXiv:1010.4309] [SPIRES].

[15] G.D. Kribs, E. Poppitz and N. Weiner, Flavor in supersymmetry with an extended R-symmetry, Phys. Rev. D 78 (2008) 055010 [arXiv:0712.2039] [SPIRES].

[16] C. Kilic, T. Okui and R. Sundrum, Colored resonances at the Tevatron: phenomenology and discovery potential in multijets, JHEP 07 (2008) 038 [arXiv:0802.2568] [SPIRES].

[17] C. Kilic, T. Okui and R. Sundrum, Vectorlike confinement at the LHC, JHEP 02 (2010) 018 [arXiv:0906.0577] [SPIRES].

[18] C. Kilic and T. Okui, The LHC phenomenology of vectorlike confinement, JHEP 04 (2010) 128 [arXiv:1001.4526] [SPIRES].

[19] D.A. Dicus, C. Kao, S. Nandi and J. Sayre, Discovering colorons at the early stage LHC, Phys. Rev. D 83 (2011) 091702 [arXiv:1012.5694] [SPIRES].

[20] J. Sayre, D.A. Dicus, C. Kao and S. Nandi, Searching for colorons at the Large Hadron Collider, Phys. Rev. D 84 (2011) 015011 [arXiv:1105.3219] [SPIRES].

[21] R.S. Chivukula, M. Golden and E.H. Simmons, Multi-jet physics at hadron colliders, Nucl. Phys. B 363 (1991) 83 [SPIRES].

[22] B.A. Dobrescu, K. Kong and R. Mahbubani, Massive color-octet bosons and pairs of resonances at hadron colliders, Phys. Lett. B 670 (2008) 119 [arXiv:0709.2378] [SPIRES]. 
[23] Y. Bai and B.A. Dobrescu, Heavy octets and Tevatron signals with three or four $b$ jets, JHEP 07 (2011) 100 [arXiv: 1012.5814] [SPIRES].

[24] A.R. Zerwekh, C.O. Dib and R. Rosenfeld, A new signature for color octet pseudoscalars at the CERN LHC, Phys. Rev. D 77 (2008) 097703 [arXiv:0802.4303] [SPIRES].

[25] A.V. Manohar and M.B. Wise, Flavor changing neutral currents, an extended scalar sector and the Higgs production rate at the LHC, Phys. Rev. D 74 (2006) 035009 [hep-ph/0606172] [SPIRES].

[26] M. Gerbush, T.J. Khoo, D.J. Phalen, A. Pierce and D. Tucker-Smith, Color-octet scalars at the LHC, Phys. Rev. D 77 (2008) 095003 [arXiv:0710.3133] [SPIRES].

[27] Y. Bai and A. Martin, Topological pions, Phys. Lett. B 693 (2010) 292 [arXiv:1003.3006] [SPIRES].

[28] T. Gleisberg et al., Event generation with SHERPA 1.1, JHEP 02 (2009) 007 [arXiv:0811.4622] [SPIRES].

[29] F. Krauss, R. Kuhn and G. Soff, AMEGIC ++ 1.0: a matrix element generator in $\mathrm{C}++$, JHEP 02 (2002) 044 [hep-ph/0109036] [SPIRES].

[30] S. Schumann and F. Krauss, A parton shower algorithm based on Catani-Seymour dipole factorisation, JHEP 03 (2008) 038 [arXiv:0709.1027] [SPIRES].

[31] A. Buckley et al., General-purpose event generators for LHC physics, Phys. Rept. 504 (2011) 145 [arXiv:1101.2599] [SPIRES].

[32] S. Hoeche, F. Krauss, S. Schumann and F. Siegert, QCD matrix elements and truncated showers, JHEP 05 (2009) 053 [arXiv:0903.1219] [SPIRES].

[33] S. Hoeche, S. Schumann and F. Siegert, Hard photon production and matrix-element parton-shower merging, Phys. Rev. D 81 (2010) 034026 [arXiv: 0912.3501] [SPIRES].

[34] T. Gleisberg and S. Höche, Comix, a new matrix element generator, JHEP 12 (2008) 039 [arXiv:0808.3674] [SPIRES].

[35] T. Carli, T. Gehrmann and S. Höche, Hadronic final states in deep-inelastic scattering with Sherpa, Eur. Phys. J. C 67 (2010) 73 [arXiv:0912.3715] [SPIRES].

[36] DO collaboration, Measurement of ratios of multi-jet cross sections in ppbar collisions at $\sqrt{s}=1.96 \mathrm{TeV}$, D0 Note 6032-CONF (2010).

[37] D0 collaboration, V.M. Abazov et al., Azimuthal decorrelations and multiple parton interactions in photon +2 jet and photon +3 jet events in ppbar collisions at $\sqrt{s}=1.96 \mathrm{TeV}$, Phys. Rev. D 83 (2011) 052008 [arXiv: 1101.1509] [SPIRES].

[38] ATLAS collaboration, J.B.G. da Costa et al., Measurement of dijet azimuthal decorrelations in pp collisions at $\sqrt{s}=7 \mathrm{TeV}$, Phys. Rev. Lett. 106 (2011) 172002 [arXiv:1102.2696] [SPIRES].

[39] A. Collaboration, Measurement of multi-jet cross sections in proton-proton collisions at a $7 \mathrm{TeV}$ center-of-mass energy, arXiv:1107.2092 [SPIRES].

[40] S. Ovyn, X. Rouby and V. Lemaitre, Delphes, a framework for fast simulation of a generic collider experiment, arXiv:0903.2225 [SPIRES].

[41] S. de Visscher, J.-M. Gerard, M. Herquet, V. Lemaitre and F. Maltoni, Unconventional phenomenology of a minimal two-Higgs-doublet model, JHEP 08 (2009) 042 [arXiv:0904.0705] [SPIRES]. 
[42] S. Ovyn, Associated $W$ and Higgs boson photoproduction and other electroweak photon induced processes at the LHC, Nucl. Phys. Proc. Suppl. 179-180 (2008) 269 [arXiv:0806.1157] [SPIRES].

[43] K. Desch, S. Fleischmann, P. Wienemann, H.K. Dreiner and S. Grab, Stau as the lightest supersymmetric particle in R-parity violating SUSY models: discovery potential with early LHC data, Phys. Rev. D 83 (2011) 015013 [arXiv: 1008.1580] [SPIRES].

[44] M. Cacciari and G.P. Salam, Dispelling the $N^{3}$ myth for the $k_{t}$ jet-finder, Phys. Lett. B 641 (2006) 57 [hep-ph/0512210] [SPIRES].

[45] M. Cacciari, G.P. Salam and G. Soyez, The anti- $k_{t}$ jet clustering algorithm, JHEP 04 (2008) 063 [arXiv: 0802.1189] [SPIRES].

[46] J. Pumplin et al., New generation of parton distributions with uncertainties from global QCD analysis, JHEP 07 (2002) 012 [hep-ph/0201195] [SPIRES]. 